\title{
Inhumane Praktiken in der I. Frauenklinik der Universität München
}

\author{
E. Kuss
}

Müchen

Es geht um nichts Geringeres als um die Weitergabe der Erinnerung im Ablauf der Generationen.

(M. Bloch, Der Brief des Historikers, 1949)

Die Schriftleitung veröffentlichte unter der Rubrik .,Zur Diskussion gestellt“ den Aufsatz ..Uber inhumane Praktiken der Frauenheilkunde im Nationalsozialismus und ihre Opfer" (Stauber und Kindermann, 1994) und schrieb dazu ein Vorwort. In diesem stellte sie fest: ..Der hier veröffentlichte Artikel will nicht anklagen und will nicht urteilen. Er will aber verdeutlichen, welch unselige Rolle der Frauenarzt spielt, wenn er zum Erfüllungsgehilfen zur Durchsetzung von politischen Ideologien degradiert wird“. Dazu nehme ich zunächst Stellung (I): im Anschluß daran zum Artikel selbst (II).

\section{1}

Die Schriftleitung stellt einen Artikel zur Diskussion, der von Zwangssterilisierungen berichtet, von Folgen des Gesetzes zur Verhütung erbkranken Nachwuchses vom 14. Juli 1933. Das Gesetz war ab 1. Januar 1934 geltendes Recht in Deutschland, Direktoren staatlicher Kliniken hatten es zu befolgen (Haselhorst, 1934). Wenn sie dadurch zu ..Erfüllungsgehilfen zur Durchsetzung politischer Ideologien degradiert" wurden, so werden es auch die heutigen Direktoren, weil und insofern sie staatliche Gesetze befolgen. Die Unterscheidung Unrechtsstaat damals und Rechtsstaat heute, mit den in beiden Fällen den jeweiligen Gesetzen immanenten Ideologien, ist im Rückblick einfach zu treffen. Es war aber kaum möglich, in der Zeit, in der am 20. Juli 1933 das Konkordat zwischen dem Deutschen Reich und der Kurie abgeschlossen wurde, in der sich die Enzyklika vom 30. Januar 1937 lediglich gegen die Kirchenpolitik wandte, in der laut Volksbefragung vom 19. August $193484 \%$, laut Reichstagswahlen vom 29. März $193699 \%$ der Wahlberechtigten der nationalsozialistischen Politik zustimmten, das Gesetz vom 14. Juli 1933 als Unrechtsgesetz eines Unrechtsstaates zu erkennen und zu mißachten.

Die Bedeutung des geltenden Rechts in der damaligen Zeit charakterisiert folgendes Zitat. .Für den Richter ist es Berufspflicht, den Geltungswillen des Gesetzes zur Geltung zu bringen, das eigene Rechtsgefühl dem autoritativen Rechtsbefehl zu opfern, nur zu fragen, was

Geburtsh. u. Frauenheilk. 55 (1995) 291-298

c) Georg Thieme Verlag Stuttgart-New York
Rechtens ist, und niemals, ob es auch gerecht sei ..." (Radbruch $^{1}$, 1932). Nach den Erfahrungen mit dem Nationalsozialismus heißt die Radbruchsche Formel: ..daß das positive, durch Satzung und Macht gesicherte Recht auch dann den Vorrang hat, wenn es inhaltlich ungerecht und unzweckmäßig ist, es sei denn, daß der Widerspruch des positiven Gesetzes zur Gerechtigkeit ein so unerträgliches $\mathrm{Ma} B$ erreicht, daß das Gesetz als „unrichtiges Recht ${ }^{\text {“ der }}$ Gerechtigkeit zu weichen hat (Radbruch, 1973). Am 5. Mai 1988 erklärte der Deutsche Bundestag das Erbgesundheitsgesetz vom 14, Juli 1933 zum NS-Unrechtsgesetz (BT Drs 11/1714).

Einer wissenschaftlichen Zeitschrift der Frauenheilkunde steht es gut an, ernsthafte fachspezifische Historiographie zu publizieren. Diese hat jedoch Tatsachen und Sachverhalte möglichst wahrheitsgetreu und vollständig und im historischen Kontext sachgerecht und zunächst wertneutral darzustellen. Eine moralisch-ethische Bewertung kann folgen oder dem urteilfähigen Leser überlassen bleiben. Jedenfalls sollte nicht schon die Wortwahl im redaktionellen Vorspann den Leser emotional auf irgendeine Tendenz einstimmen.

\section{II}

Die Autoren des von der Schriftleitung zur Diskussion gestellten Artikels sind Professor Dr. $M$. Stauber, gegenwärtig leitender Oberarzt, und Professor Dr. G. Kindermann, gegenwärtig Direktor der I. Frauenklinik der Universität München. Die Autoren erörtern unter dem generalisierenden Titel hauptsächlich ausgewählte Einzelheiten aus der Geschichte dieser Klinik, solche aus der Zeit 1933-1945. als Professor Dr. A. Döderlein und anschließend Professor Dr. H. Eymer Direktoren der Klinik waren, und ausgewählte Einzelheiten aus der Biographie

\footnotetext{
"Die unter der Rubrik ,.zur Diskussion gestellt" veröffentlichten Beiträge, müssen nicht unbedingt mit den Auffassungen der Schriftleitung übereinstimmen.

1 Prof. Dr. Dr. h. c. Gustav Radbruch. 1878-1949; Sozialdemokrat, 1920-1924 Mitglied des Reichstages, 1921-1923 Reichsjustizminister: Rechtslehrer verschiedener deutscher Universitäten, 1933-1945 amtsenthoben (Kaufmann, 1987).

Prof. Dr. Dr. h. c. Hans Nachtsheim. 1890-1979; Kaiser-Wilhelm-Institut für Anthropologie, menschliche Erblehre und Eugenik. Max-Planck-Institut für vergleichende Erbbiologie und Erbpathologie; 1961 Berater im Wiedergutmachungsausschuß zur Entschädigung der im Nationalsozialismus gegen ihren Willen Sterilisierten; gehörte keiner NS-Organisation an (Deichmann, 1992).
} 
Eymers. Mit diesen Einzelheiten vermengen die Autoren zeittypische Probleme der Medizin. die als Oberschätzung humangenetischer Kenntnis und eugenischer Möglichkeiten, als Konflikt zwischen Individual- und Sozialmedizin. als Obergang von kurativer zur präventiven Medizin gesehen wurden und werden, und Verbrechen anderer, die auch damals eindeutig als solche erkennbar waren. Die Autoren suggerieren, daß Fragen der Vergangenheit mit den Antworten der Gegenwart hätten gelöst werden können. Sie argumentieren mit unscharfen Begriffen und Redensarten und bemühen sich mehr darum, Betroffenheit nachzuweisen als Sachkenntnis. Jedoch, von anderem abgesehen, allein schon die Zusammenfugung zweier disparater Begriffe im Titel des Artikels, nämlich ..inhumane Praktiken" einerseits und ..Opfer" andererseits, läßt gerade die Sensibilität vermissen, die sich die Autoren zugutehalten (hierzu s, z. B. Wuttke, 1992), und läßt die Art Psychosomatik, auf die sie sich berufen, als sei sie eine Art besserer Medizin, eher fragwürdig erscheinen. Die Quellenangaben im Text sind in erheblichem Maße defizitär. ein Großteil der Literaturzitate ist wenig sachdienlich.

Zur Verurteilungsabstinenz der Autoren. Eymer konnte sich zu seinen Lebzeiten mit Zeugen seiner Zeit gegen ehrenrührige Vorwürfe wehren. Dies ist ihm heute nicht mehr möglich. So kann er postum von den Autoren Stauber und Kindermann des ..Verbrechens gegen die Menschlichkeit" bezichtigt werden und von ihnen vorgeführt werden als einer, der nicht würdig war, von der Bayerischen und Deutschen Gesellschaft für Gynäkologie und Geburtshilfe zum Ehrenmitglied ernannt und von der Bundesrepublik mit dem großen Verdienstkreuz zum Verdienstorden ausgezeichnet zu werden. Es stellt sich die Frage, warum darüber keine Stellungnahmen der genannten Gesellschaften und des Bundespräsidialamtes vorliegen. Die Autoren fällen mehrfach ihre Urteile über Eymer (s. auch Anhang 1 und 2). Durch entsprechende Akzentuierungen und Textmontagen wie auch durch mangelhafte Strukturierung und Präzisierung ihrer Aussagen legen die Autoren ihren Lesern die Verurteilung Eymers nahe. So reduzieren sie die Glaubwürdigkeit ihres Bekenntnisses zur ..Verurteilungsabstinenz". Dies bestätigen auch die Kommentare von Journalisten der Laienpresse wie .Eine späte Entschuldigung- (Süddeutsche Zeitung vom 31.8.1994) und ..Kaum faßbare ärztliche Inhumanität" (Frankfurter Allgemeine Zeitung vom 7.9.1994)

Zur Vergangenheitsbewailtigung der Autoren. Kein Mensch kann die Vergangenheit anderer Menschen .,bewältigen”, die eigene Gegenwart ist zu bewältigen (s. a. Anhang 1 u. 2). Was immer man unter ..Vergangenheitsbewältigung " verstehen mag. sie setzt Kenntnis oder Erforschung des Vergangenen voraus. Diese Forschung braucht, wie jede andere Forschung, mühsame Kleinarbeit mit hinreichender Fachkompetenz und Redlichkeit im Umgang mit den Ergebnissen. Ich zweifle daran. daB beim Abfassen des zur Diskussion gestellten Artikels diese Voraussetzungen immer erfült wurden.

In ihrer .,Vergangenheitsbewältigung" behandeln die Autoren Stauber und Kindermann den Begriff ,Generation“, eine heuristische Hypothese der Soziologie ( $K$. Mannheim. 1928), wie eine Tatsache und stellen generationenspezifische Verhaltensmuster als reale soziale Kategorien dar: Vater ,der Täter“ - Sohn ,.des Täters Beschützer" - Enkel „der vom Täter Freie/Befreite/Befreiende* (Kurzassung von mir). Allein der Hinweis auf Mitscherlich und Mielke sollte die Schwäche dieses Ansatzes kennzeichnen, auch die Genealogie der Autoren, zumindest die des Autors GK, stützt ihn nicht. Auch folgendes paßt nicht in das Schema: .Fs ist ein Unding, das nach dem Vorbild der Gesetzgebung von Kulturstaaten geschaffene Erbkrankheitsgesetz fur die Naziuntaten auf dem Gebiet der Rassenpolitik und Euthanasie verantwortlich zu machen." Der Jurist Neukamp, der sich u. a. hiermit 1951 gegen die damals von der Arbeitsgemeinschaft sozialistischer Ārzte angestrebte ..Wiedergutmachung" der sterilisierten Ërbkranken wandte (Anfuhrungsstriche vom zitierenden Autor), war offenbar weder ..Täter" noch „Sohn eines Täters" sondern rassisch Verfolgter (Nachtsheim 1 1962), der sich 1937 offentlich gegen die Euthanasie ausgesprochen hatte (Schmuhl, 1987, S. 179). Stauber und Kindermann vernachlässigen auch, daB Leibbrand. Matussek und Mitscherlich wahrlich nicht zu den ..Enkeln“ gehören. Diese Mediziner lehnten 1963 das „Gesetz. zur Verhütung erbkranken Nachwuchses" von $1933 \mathrm{ab}$ und auch die aktuellen Vorschläge des Humangenetikers Nachtsheim für ein neues Erbkrankheitsgesetz. Sie begründeten ihre Ablehnung im wesentlichen mit der „nach Auschwitz" veränderten allgemeinen Bewußtseinslage, aber die moralische Integrität Nachtsheims zweifelten sie nicht an (Leibbrand. 1963: Matussek, 1963; Mitscherlich. 1963. Nachtsheim, 1963). Auch verträgt es sich nicht mit der Generationenhypothese von Stauber und Kindermann. daß sich 1987 der Kanzleramtsminister W. Schäuble (Jahrgang 1942) gegen eine Entschädigung für Zwangssterilisierte aussprach. wogegen der Direktor einer Klinik fur Psychiatrie, Psychosomatik und Neurologie, K. Dörner, (Jahrgang 1933) für eine Entschädigung für Zwangssterilisierte plädierte und maBgeblichen Anteil daran hatte, daß es zu der einschlägigen Entschließung des Bundestages kam (BT Drs 11/1714: Forum, 1987; Dörner. 1993). Allerdings schreibt auch Dörner hinsichtlich der historischen Entwickung hin zur Sterilisierung Behinderter und zur aktiven Sterbehilfe ...Mit Sicherheit konnten unsere psychiatrischen Vảter und Großväter, die um 1940 herum Verantwortung zu tragen hatten, diesen Zusammenhang kaum erkennen." (Dörner 1989).

Im folgenden gehe ich auf nur einige der Aussagen der Autoren Stauber und Kindermann ein. die ich für fragwürdig halte. Ich beschränke mich auf den Bereich Eymer und Zwangssterilisierung, auf den auch sie ihre Aufmerksamkeit fokussiert haben. Wie die Autoren feststellten, ist ausschließlich .Zwangssterilisierung" der Bereich, für den Eymer inhumaner Praktiken beschuldigt wird. Um gewohnte Unterstellungen von vornherein zu unterbinden, sei unmißverständlich erklärt: Meine Sache ist nicht, Eymer zu exkulpieren, die Zwangssterilisierung zu propagieren oder gar den Nationalsozialismus zu bagatellisieren. Meine Sache ist, die von der Redaktion angebotene Diskussion des Artikels von Stauber und Kindermann ..Uber inhumane Praktiken der Frauenheilkunde im Nationalsozialismus und ihre Opfer" aufzunehmen.

1. Die Autoren behaupten, daB Eymer ,in der Gerichtsverhandlung wegen seines Verhaltens in der Nazizeit 
... aus dem Amt entfernt" wurde. Dies entspricht nicht den Tatsachen. In der amerikanischen Besatzungszone wurden Angehörige des öffentlichen Dienstes auf Befehl der Militärregierung nach formalen Kriterien, z. B. Zugehörigkeit zur NSDAP, kollektiv aus dem Amt entfernt. Anschließend wurde in sogenannten Spruchkammern über jeden Fall einzeln und in Würdigung seiner persōnlichen Umstände befunden (Buchheim. 1993). Auch Eymer wurde auf Weisung der Militärregierung entlassen und mußte sich einem Spruchkammerverfahren unterziehen (s. die folgenden Anmerkungen 2. u. 3.J Aufgrund der für ihn positiven Beweislage in Sachen Zwangssterilisierung und der fur ihn positiven Zeugenaussagen über sein Verhalten zum NS-Regime kam es .in der Gerichtsverhandlung" zu seiner weitgehenden Rehabilitierung (Einstufung als ,.Mitläufer“). So konnte er unmittelbar darauf, April 1948 (und nicht ..1949"), erneut in sein Amt berufen werden, das er dann bis 1954 ausübte. Dieser Sachverhalt ist neben weiteren Einzelheiten auch in der vom Autor MS betreuten Dissertation (Kettler, 1994) korrekt beschrieben worden.

2. Die Autoren stellen die Mitgliedschaft Eymers in NSOrganisationen und schlicht zeitübliche Formulierungen in offiziellen Briefen heraus, obwohl dies nicht notwendigerweise zum Thema gehört. Außerdem. selbst wenn über Eymers Mitgliedschaft u. ä. Dokumente vorliegen, zu ihrer Deutung im Rahmen der Geschichtsschreibung gelten Vorbehalte, auf die kürzlich in der Zeitschrift .Psyche" ausfuhrlich und mit Hinweisen auf maßgebliche Historiker der Gegenwart wie Bloch und Le Goff eingegangen wurde (Bloch. 1949), Oder man kann auch einfach bedenken .Als Kurt Huber sich 1940 zur Aufnahme in die NSDAP bewarb, dürfte er innerlich bereits von dem NS-Staat soweit entfernt gewesen sein wie nie zuvor. ...- $(R$. Wiehl. 50. Jahrestag der Hinrichtung von Prof. Dr. Kurt Huber und Alexander Schmorell, in: Chronik der Ludwig-Maximilians-Universität 1991-1993). Die Welt ist nicht so einfach und klar, daß derjenige, der sich dem Gesetz zur Verhütung erbkranken Nachwuchses nicht widersetzte. sondern als Staatsdiener das Gesetz befolgte, oder Zwangssterilisierungen Erbkranker als eine Möglichkeit zur Minderung menschlichen Leids oder zur Realisierung platonischer Staatsutopie anerkannte oder gar Mitglied einer NSOrganisation wurde, schon deswegen ein schlechter Mensch, Synonym ..Nazi“, war (s. Anhang 2). Die Autoren Stauber und Kindermann fanden das Verhalten der katholischen Ordensschwestern .Als einzigen Nachweis für einen Widerstand gegen nationalsozialistische Anordnungen". Bekanntlich sind aber die Arten und Motive des ..Widerstandes" gegen den Nationalsozialismus ähnlich vielseitig gewesen und somit ähnlich schwierig zu beurteilen wie die Arten und Motive seiner „Förderung". Die Autoren behaupten. daß aus Eymers „späterem Schriftverkehr mit dem Reichsministerium des Inneren sowie seiner Stellungnahme zu Bitten von Patientinnen zwecks Verschonung von solchen Zwangsmaßnahmen" seine Unterstūtzung der ..Idee der Rassehygiene* besonders deutlich wird. Sie gehen nicht auf die sehr unterschiedlichen Begriffsinhalte von „Rassenhygiene“ ein. die von ..Verbesserung (oder Vermeidung der Ver- schlechterung, der .Degeneration") ..der menschlichen Rasse“ (des ..Genpools der Specie Mensch" in heutiger Diktion), über die vermeintliche Minderwertigkeit jedweder Art ..rassischer Mischlinge" und der Höherwertigkeit einer, z. B. der ..arischen Rasse“. bis hin zur .Ausmerzung", zur physischen Vernichtung .Fremdartiger" reichen (s. z. B. Ritter, 1992; Schmuhl, 1987). Statt dessen zitieren sie die vom Autor MS betreute Doktorandin. In ihrer Doktorarbeit findet sich aber über den Schriftverkehr nichts und als Stellungnahme Eymers allein folgendes: ..... Da hier aber Sicherheit gefordert werden muB, so ist eine Unfruchtbarmachung meiner Auffassung nach bei Frau $H$. noch angezeigt. sofern diese durch das Grundleiden gefordert wird. ... (Kettler. 1994. Hervorhebung von mir). In dieser einzigen zitierten Stellungnahme Eymers kann ich keine besonders deutliche Unterstützung der „Idee der Rassehygiene”, insbesondere nicht die der NS-Rassenhygiene erkennen, die aus heutiger Sicht „Endlösung Auschwitz” bedeutet (s. Anhang 2). Außerdem schreibt die Doktorandin in dieser Dissertation, daß als Pressemitteilung zum Spruchkammerverfahren berichtet wurde, daß ..Prof. Eymer in vielen Fällen beweisen konnte, daß er sich gegen den nationalsozialistischen Geist in der Klinik. soweit es möglich war. gewehrt hatte." (Kettler, 1994).

3. Die Autoren lassen die Aussagen über Eymer unberücksichtigt, die von ihrer offenbar vorgefaßten Meinung über ihn abweichen. Beispielhaft für die den Autoren bekannten positiven Äußerungen über Ey. mer sind die

- des verstorbenen Professors Dr. Rolf Kaiser. Zeuge im Spruchkammerverfahren gegen Eymer und Schüler Eymers (Brief vom 31.3.1992 an den Autor GK).

- des Herrn Dr. R. Haselwarter, Doktorand unter Eymer (Zitat Nr. 19 und 20 der Autoren), im Seminar des Autors MS am 19. Juli 1994 (R. Haseltwarter. persōnliche Mitteilung).

- des Professors Dr. Fritz Zimmer (Briefe vom 13. Januar und 6. April 1992 an den Autor MS).

- von mir mit Zitat aus Sr. M. Leodegar ..Meine Erinnerungen aus der Frauenklinik von 1927-1975" (Brief vom 6.3.92 an den Autor GK). Die zitierte schriftliche Aussage der Ordensschwester: - für Fy. mer, ..der kein .Nazi " " war, ist es oft nicht leicht gewesen, mit heiler Haut davonzukommen, da einige junge Assistenten sehr für den ..Führer" waren", - steht im Widerspruch zu der Behauptung der Autoren über Gespräche ,mit einigen Schwestern dieser Zeit”, sofern deren ,belastende Hinweise" auf Eymer gemünzt gewesen sein sollten.

- von der Doktorandin in ihrer vom Autor MS betreuten Dissertation. Ihr oben (s. 2) aufgeführtes Zitat (Kettler. 1994) ist praktisch identisch mit dem der $\operatorname{Sr}, M$. Leodegar über Eymer.

Nach allem, was ich von den ärztlichen Kollegen und von den barmherzigen Schwestern, die mit Eymer gearbeitet haben, über Eymer erfahren konnte, hat er einen Biographen verdient, der seine Verdienste um die 1. Frauenklinik der Universität München, um die Abschirmung ihrer katholischen Ordensschwestern gegen NS-Willkür, um die Behandlung ihrer Patienten. die Ausbildung ihrer Studenten und um die Weiterbil- 
dung ihrer Ärzte darstellt und sowohl seine Verstrikkung möglicherweise auch in persönliche Schuld wie auch seinen Streit mit der ärztlichen NS-Fraktion der Klinik mit Noblesse und Sachverstand beschreibt. Eymer übernahm mit der Münchner Frauenklinik vom Vorgänger als Oberarzt einen ..Träger des Blutordens (s. z. B. Kater, 1989, S. 127-129), ein SS-Hauptsturmführer, komissarischer NS-Gau-Dozentenbundführer und NS-Dozentenfürer der Universität München und andere, der Klinik vom NS Studentenbund zugewiesene Ärzte gehörten seinerzeit zum Kollegium und kooperierten mit übergeordneten Parteistellen. Bei Kenntnis dieser Randbedingungen sollte man ermessen können. was es heißt, „daß er sich gegen den nationalsozialistischen Geist in der Kinik, soweit es möglich war, gewehrt hatte. (Kettler, 1994). Auch die formale Mitgliedschaft in NS-Organisationen könnte als Selbstsicherung um dieser Abwehr willen erfolgt sein. Ober diese naheliegenden Denkmöglichkeiten und ihre Belegbarkeit durch historische Fakten findet sich im Artikel von Stauber und Kindermann kein Wort, trotz einschlägiger Aussagen in der vom Autor MS betreuten Dissertation (Kettler. 1994). So entsteht der Eindruck, daß die Autoren Stauber und Kindermann nicht um der historischen Wahrheit willen „ein nun einmal beschmutztes Nest". wie sie es nennen. beanspruchen.

4. Die Autoren stellen das Nicht-Mitwirken katholischer Ordensschwestern bei Sterilisierungen (hierzu s. auch Anhang 1) als Beispiel für den Widerstand der Schwestern dar und als Gegenbeispiel zum Verhalten $E y$ mers. Das Abtreten von Ordensschwestern und von anderen bei einschlägigen Operationen, zum Beispiel Abtreibungen, war auch noch in meiner Klinikzeit (ab 1960) üblich. Könnte es nicht möglich sein, daß auch in der NS-Zeit die Schwestern im Konsens mit dem Klinikdirektor handelten, der möglicherweise die Haltung der Schwestern und ihre Vorstellung von Sittlichkeit respektierte? Aber auch die juristische Grundlage der jeweiligen Verhaltensweise wird von den Autoren Stauber und Kindermann vernachlässigt. Eymer war als nichtkatholischer Staatsbeamter zur Durchführung der gesetzlichen Sterilisierung verpflichtet (Haselhorst. 1934), die Ordensfrauen nicht. Die katholische Kirche hatte bereits mit der Enzyklika vom 13.12.1930 dem Staat das Recht zu eugenisch begründeten Sterilisierungen abgesprochen. Aufgrund von Sonderregelungen zwischen Episkopat und Reichsregierung waren katholische Ärzte, Schwestern und Richter von Teilen der Sterilisierungspraxis ausgenommen. Die Bischofskonferenz untersagte am 6. Juni 1934 Ordensleuten die Mitwirkung bei Sterilisierungen. Die evangelische Kirche, Innere Mission, war bereits 1929/1931 für Sterilisierungen eingetreten (Literatur S.z.B. Schmuhl, 1987. S. 305; Dörner. 1988, 1993).

5. Die Autoren suggerieren, das zumindest damals real existierende Problem .Eugenik" einfach dadurch zu lösen, ..den inhumanen Anfängen von ärztlicher Seite entsprechend dem Hippokratischen Eid zu widerstehen". Was sind und wie erkennt man prospektiv inhumane Anfänge? Der sogenannte Hippokratische Eid taugt doch offenbar nicht zur Lösung realer medizinischer Probleme. Der Hinweis auf das Kapitel „Ratio- nierung" in der Eröffnungsansprache des Präsidenten der Deutschen Gesellschaft für Gynäkologie und Geburtshilfe (Hepp. 1995) müßte als Begründung genügen, denn es sollte bekannt sein, da $B$ „Rationierung“ die utilitaristische Begründung der Zwangssterilisierung konstituierte, (Zu Hippokrates s. auch z. B. Geschwandtner-Andre $\beta$, 1993: Der Hippokratische Eid, keine normative Richtschnur für ärztliches Handeln vor dem gewandelten wissenschaftlichen und sozialen Kontext der Gegenwart). Und "The evils properly associated with eugenics have caused us to forget that it did have a partly rational basis.." (Van Valen, 1989). Der Vorrang der Selbstbestimmung des Individuums vor der Fremdbestimmung durch ..Staat". ...Kirche" oder ..Klasse", auch in Fragen der Reproduktion, ist eine relativ neue und relativ lokale Errungenschaft. die auch hier und jetzt widersprüchlich gehandhabt wird. Die Entscheidung des Arztes im Konfliktfall für seinen" Patienten gegen ..seine- Gesellschaft (auch gegen die..Solidargemeinschaft"?) gilt als hippokratisches Erbe, ist aber ethisch nicht zwingend geboten (Verkürzte Begründung: Joh. 11. 50). Außerdem wird auch die aktuelle ärztliche Ethik der westlichen Welt, und somit auch die der Autoren Stauber und Kindermann, von der Jusrisdiktion dominiert (Tauber. 1993: Burkart 1994).

6. Die Autoren werfen A. Döderlein und seinem Nachfolger $H$. Eymer vor, jeweils die Artikel .Die (Eingriffe zur) Unfruchtbarmachung der Frau für die 1. (1934) respektive 2. Auflage (1936) von Gütt/Rüdìn/Wuttke ..Gesetz zur Verhütung erbkranken Nachwuchses“ geschrieben zu haben. Beide Artikel behandeln ausschlieBlich die gynäkologischen Probleme des Eingriffs und der Nachsorge. Ist es nicht so, daß es eine - wie auch immer geartete und teilweise von der zeitbedingten öflentlichen Meinung abhängige - Indikation zur Sterilisierung gab und gibt, und daB es ärztliche Aufgabe war und ist, den dazu notwendigen Eingriff kompetent zu beschreiben und dadurch innerhalb des jeweiligen EinfluBbereiches so risikoarm zu gestalten wie möglich? Es besteht doch die Möglichkeit, daß die angegebene Mortalitätsrate von $0,46 \%$ für intraperitoneale Eingriffe beim damaligen Stand der Medizin als gering angesehen werden muß (was ich nicht beurteilen kann), daß dies dem oben genannten Artikel Eymers zu verdanken war, und daB ein Teil der heute zur „späten Entschuldigung "2 herangezogenen Frauen just diesem Artikel ihr Leben zu verdanken haben. Ist Eymer nicht eher zu loben, wenn er, wie die Autoren Stauber und Kindermann behaupten, eine Methode zur Sterilisierung, die auch heute noch zur Konzeptionsverhütung gängig ist. ..perfektionierte"? Ist es heutigen Gynäkologen fremd, daß ein „Eingriff nicht selten operative Schwierigkeiten brachte"? Wird Eymer etwa auch die Tatsache zur Last gelegt, daß er bereits den Film als didaktisches Hilfsmittel heranzog?, daß er über seine operative Tätigkeit in klaren Statistiken Rechenschaft ablegte?

2 Diese Anwendung des Begriffs ..Entschuldigung" durch die Autoren ist fragwürdig: Im allgemeinen steht im Deutschen „entschuldigen" für ..positiv rechtfertigen", ...ich mußte dies tun, um eine andere und wichtigere Pnicht zu erfullen" (Schiller, 1980. S. 223. 
Die Autoren unterlassen es, darauf hinzuweisen, da B zwischen den von Döderlein und Eymer vertretenen Methoden zur Unfruchtbarmachung von Frauen und den von Clauberg entwickelten Methoden zur Massensterilisation (Grosch. 1985) konzeptionelle Unterschiede bestehen.

7. Die Autoren berichten ..über 1000 Patientinnen mit Zwangssterilisierung und z.T. Schwangerschaftsabbruch gegen den eigenen Willen ${ }^{-}$in der L. Frauenklinik der Universität München, ohne auch nur einen ..Schwangerschaftsabbruch gegen den eigenen Willen" aufzufuhren (bei, laut Tabelle, insgesamt $9 \mathrm{Ab}$ brüchen). Bekanntlich wurde der Schwangerschaftsabbruch bei Frauen. die ein als erbkrank eingestuftes Kind erwarteten, in der NS-Praxis sehr unterschiedlich gehandhabt (Schmuhl, 1987, S. 162 ff.). Zumindest nach dem Text der vierten Verordnung zur Ausführung des Gesetzes zur Verhütung erbkranken Nachwuchses vom 18. Juli 1935 war die Einwilligung der Schwangeren Voraussetzung zum Abbruch. Eymer selbst schrieb dazu unmißverständlich .Die Unterbrechung kann nur mit Einwilligung der Schwangeren oder ihres Vormundes durchgeführt werden." (Eymer, 1936). Im Spruchkammerverfahren von 1947 wurde Eymer zugute gehalten ,.daß jeder an der Klinik durchgeführten Zwangssterilisation entweder eine Einwilligung der Frau oder ein Beschluß des Erbgesundheitsgerichts zugrunde lag", was in der vom $\mathrm{Au}$ tor MS betreuten Dissertation zitiert worden ist (Kettler. 1994).

8. Die Autoren haben von den tausend Fällen von Zwangssterilisierung in der L. Frauenklinik der Universitắt München zwei Kasuistiken ausgesucht (rachitische Hände, Glaukom), die Eymer offenbar besonders belasten sollen. Sie stellen das Schicksal zweier Frauen vor, deren Sterilisierung selbst nach dem .,Gesetz zur Verhütung erbkranken Nachwuchses" nicht legal war. ohne daß die Autoren nachweislich erforscht haben, wie das Gutachten des ..Erbgesundheitsgerichts" formuliert war und zustande gekommen ist. Die Indikation zur Sterilisierung wurde durch dieses Gremium gestellt und nicht durch den operierenden Arzt, wie auch das oben (7.) aufgefuhrte Zitat aus der genannten Dissertation bestätigt (Kettler. 1994; s. a. Rothmaler, 1989). Außerdem, auch heute sind Indikationen zu Operationen nicht absolut frei von Irrtümern. Die Autoren Stauber und Kindermann beanstanden, daß laut Krankengeschichten in der Klinik Eymers $16 \%$ der Sterilisierten ..ohne genauere Diagnose" - aber immerhin mit dem Vermerk ..Gesetzliche Sterilisation" - operiert worden sind. Aber unterscheiden sich denn diese Fehler in der Dokumentation kategorial von denen, die im Alltag deutscher Universitäts-Frauenkliniken unserer Zeit gemacht werden? Nach meinen Erfahrungen unterscheiden sie sich nicht. Ähnliches gilt für die von Stauber und Kindermann behauptete Selbstverständlichkeit, heutzutage ...̈rztliche Behandlungen durch nachgehende Fürsorge, ein ..follow up" zu überprüfen, zu bewerten und Erkenntnisse und Schlüsse aus dem zurückliegenden Vorgehen zu gewinnen". Dies ist meines Erachtens noch immer keine Selbstverständlichkeit.
9. Die Autoren bringen die tödlichen Komplikationen nach Zwangssterilisierung in der 1. Frauenklinik der Universität München $(0,46 \%)$ in die Nähe der Tötung von Behinderten, der Selektion und der Tötung von KZ-Häftlingen durch sogenannte wissenschaftliche Experimente am Menschen. Daß deutsche Ärzte an diesen Vorgängen maßgeblich beteiligt waren, dürfte ebenso allgemein bekannt sein wie ihre zeitliche $\mathrm{Ab}$ folge, ihre sehr unterschiedlichen Rechtsgrundlagen. Bekanntheiten und Akzeptanzen im In- und Ausland. Die hieraus resultierenden Differenzierungen fuhrten dazu, daB 1946 im Nürnberger ÄrzteprozeB die Zwangssterilisierung nach dem ..Gesetz zur Verhütung erbkranken Nachwuchses" nicht verhandelt wurde, wohl aber die Verbrechen an Behinderten und Häftlingen. Bekanntlich führten andere Länder die Zwangssterilisierung ein, ohne da $\mathrm{B}$ sie zwangsläufig auch .Auschwitz" errichteten (über ideelle Divergenzen zwischen Eugenik und Euthanasie in Deutschland s. Schmuhl, 1987, S. 178; über institutionelle Konvergenzen von Euthanasie und Holocaust s.u. a. M. Broszat. H. Mommsen, zitiert bei Schmuhl, 1987, S. 365). Wenn aber der Sterilisierung keine Vorläuferfunktion mit Euthanasie und Holocaust als zwingende Folgen zuzuweisen ist, muß auch Eymer und der I. Frauen. klinik der Universität München die entsprechende Einzelfallgerechtigkeit gewährt werden. und die Diskussion muB sich fairerweise und historisch korrekt auf das Gebiet der Sterilisierung aus eugenischer Indikation beschränken, wie es auch die Autoren anfãnglich einräumten. Warum bleiben sie nicht dabei und berichten über Eymer ähnliches wie das, was über Karl Bonhoeffer gesagt wurde, .the nestor of german psychiatrists, who condemned medical killing but does not appear to have opposed sterilization with any consistence, if at all.") (Kater, 1989, S. 146). Zu den Eymer ebenfalls mehr oder weniger deutlich angelasteten Maßnahmen zur Erzwingung der gerichtlich angeordneten Sterilisierung:

a) jedwede Gesetzeskraft, auch die des Rechtsstaats, ist notwendigerweise mit zwingender Gewalt verbunden und

b) Devianzen und ihre medizinische Zwangsbehandlung wurden auch schon vor 1933 anders beurteilt als nach 1945 oder gar nach 1990.

c) Eymer wurde keine Gewaltanwendung nachgewiesen, auch keine entsprechende Anweisung.

10. Die Autoren haben für ihren Abschnitt „Ein Blick von draußen" ein extrem eingeschränktes Blickfeld ausgewählt. Sie gehen weder auf ausländischen Applaus für das ..Gesetz zur Verhütung erbkranken Nachwuchses" ein noch auf die zunächst außerhalb Deutschlands erfolgte Entwicklung der Eugenik mit Zwangssterilisierung in Theorie (F. Galton, 1822-1911, GB) und Praxis (Indiana. 1907, USA) (s. z. B. Garver und Garver, 1991). Auf diesen Aspekt hat jetzt ein deutscher Historiker (Kühl, 1994) erneut hingewiesen und auch ein Publizist: ..Nicht ohne Schreck liest man nebenher, $\mathrm{da} B$ ein britischer Innenminister namens Winston Churchill ernstlich vorhatte. über 100000 Menschen zwangssterilisieren zu lassen." (Janßen, 1994). Doppelt unredlich erscheinen mir die Ausführungen des Abschnitts, weil sie den Eindruck erwekken kōnnen, als würden die ..von draußen" deutsche 
und nationalsozialistische Vorurteile über das Sein anderer Nationen und Rassen mit umgekehrtem Vorzeichen weiter pflegen. Erstaunlich, daB die Autoren Stauber und Kindermann ausgerechnet hier Hitler. „Mein Kampf”, zitieren und hier die Frage finden, „ob es nicht eine spezifische deutsche Struktur gibt, die es möglich machte, eine solche Diktatur zu entwickeln“. Sollten aber auch die Autoren Stauber und Kindermann der Meinung sein, daß Äußerungen dieser Art für das Ausland eher atypisch sind, warum dann dieses meines Erachtens gefährliche Spiel mit nationalen Etikettierungen und .Erbsträngen“ und der abstrusen Feststellung, daß ,die Liebe zum Kind wohl keine germanische Tradition habe“?

Der Deutsche Bundestag hat am 5. Mai 1988 ausdrücklich beschlossen, daß u. a. die Krankenanstalten die noch lebenden Zwangssterilisierten gezielt auf Entschädigungen hinweisen sollen (BT Drs, 11/1714) ${ }^{3}$. Es hätte also nicht erst des von den Autoren genannten Anlasses der Weihnachtsfeier 1991 und des 75jährigen Bestehens der I. Frauenklinik der Universität München (ihr Zitat 64) bedurft, um dieser Aufgabe nachzukommen. Meines Erachtens wäre es für die Autoren Stauber und Kindermann, für ihre Leser und für das Ansehen ihrer Klinik besser gewesen, sie hätten nach den Gepflogenheiten medizinisch-wissenschaftlicher Publizistik über konkrete eigene Erfahrungen berichtet, zum Beispiel über die Indikationen zur gynäkologischen Behandlung derer, die infolge des vom deutschen Volk mehrheitlich gebilligten oder billigend in Kauf genommenen Gesetzes zur Verhütung erbkranken Nachwuchses geschädigt wurden und über die Ergebnisse dieser Behandlungen. Mit den Worten der Autoren: „Aus psychosomatischer Sicht muß die Erinnerung konkret sein, um Betroffenheit auslösen zu können und Trauer zu ermöglichen. Dies geschieht am wirkungsvollsten im eigenen Arbeitsbereich." Die Beteuerung der eigenen Betroffenheit, Sensibilität und edukatorischen Effizienz kann wissenschaftlich kompetente und sorgfältige Forschung und korrekte Darstellung ihrer Ergebnisse nicht ersetzen.

\section{Literatur}

Bloch, M.: Der Brief des Historikers, 1946, zitiert nach Steiner. R,: ..Es ist eine neue Art von Diaspora". Psyche 43 (1994) 583-652; dort auch Referenz zu J. Le Goff.

Buchheim. H.: Zur öffentlichen Auseinandersetzung mit unserer nationalsozialistischen Vergangenheit. In Benz. W. . H. Buchheim, H. Mommsen (Hrsg.): Der Nationalsozialismus. Fischer Taschenbuch Verlag, Frankfurt/M. (1993).

Burkart, W.: Ethik contra Gesetz? Deutsches Ärzteblatt 91 (1994) C-1035.

Deichmann, U.: Biologen unter Hitler. Vertreibung, Karrieren. Forschung. Campus-Verlag, Frankfurt, New York (1992).

Dörner. K.: Was unterscheidet die heutigen Oberlegungen zur Sterilisation von Menschen mit geistiger Behinderung von der Zwangssterilisation der NS-Zeit? Spektrum der Psychiatrie und Nervenheilkunde 17 (1988) 64-73.

Dörner, K.: Anstaltsalltag in der Psychiatrie und NS-Euthanasie. Deutsches Ärzteblatt 86 (1989) B-534-538.

Dörner, K.: Tödliches Mitleid. Zur Frage der Unerträglichkeit des Lebens oder: die Soziale Frage: Entstehung, Medizinierung,

\footnotetext{
3 siehe auch WZ: Dt. Ärztebl. 87 (1990) A-3708 ..Härteleistungen für sterilisierte NS-Opfer"
}

NS-Endlösung heute morgen. Verlag Jakob von Hoddis, Gütersloh (1993).

Eymer, H.: Die Unfruchtbarmachung der Frau: In Gütt, A., E. Rüdin, F. Ruttke: Gesetz zur Verhütung erbkranken Nachwuchses. J. F. Lehmanns Verlag. München (1936).

Forum: Vergessene Opfer des NS-Regimes. Spektrum der Psychiatrie und Nervenheilkunde 16 (1987) 72

Garver, K. L, B. Garver: Eugenics: past present and the future. Am. J. Hum. Genet. 49 (1991) 1109-1118.

Geschwandtner-Andreß. P.: Fast 2400 Jahre alt und noch immer im Gespräch: Der Hippokratische Eid. Deutsches Ärzteblatt 90 (1993) B-2487-2488.

Grosch. H.: Carl Clauberg (1898-1957), ein biographischer Hinweis. Endokrinologie-Informationen 9 (1985) 103-108.

Haselhorst, G.: Zur Sterilisierung der Frau aus eugenischer Indikation. Deutsche Medizinische Wochenschrift (1934) 14301432.

Hepp. H.: Zwei Leben - Anspruch und Wirklichkeit. Vorabdruck Arch. Gynecol. 257 (1995) (Erweiterte Fassung der Eröffnungsansprache vom 23. August 1994).

Janßen, K.-H.: Kunst der Auslese. Die Zeit, 1,4. 1994.

Kater, M. H.: Doctors under Hitler. The University of North Carolina Press, Chapel Hill and London (1989)

Kaufmann, A.: Gustav Radbruch - Rechtsdenker, Philosoph, Sozialdemokrat. R. Piper, München (1987).

Kettler, K.: Aspekte zur Geschichte der I. Universitäts-Frauenklinik München von 1916 bis 1945. Dissertation, Medizinische Fakultät der Ludwig-Maximilians-Universität München (1994).

Kühl, S.: The Nazi Connection. Eugenics, American Racism and German National Socialism. Oxford University Press, New York, Oxford (1994).

Leibbrand, W.: Eugenik und Sterilisation. Fortschritte der Medizin 81 (1963) 718.

Mitscherlich, A.: Eugenik - Notwendigkeit und Gefahr. Fortschritte der Medizin 81 (1963) 714-715.

Mannheim, K.: Das Problem der Generationen, zitiert nach $\mathrm{H}$. Bude, Deutsche Karrieren. Suhrkamp, Frankfurt (1987).

Matussek, P.: Gedanken eines Psychiaters zur Frage der gesetzlichen Sterilisation. Fortschritte der Medizin 81 (1963) $716-$ 718.

Nachtsheim. H.: Das Gesetz zur Verhütung erbkranken Nachwuchses aus dem Jahre 1933 in heutiger Sicht. Ärztl. Mitteilungen 47 (1962) 1640-1644; Aussprache: 2515-2519.

Nachtsheim, H.: Warum Eugenik? Fortschritte der Medizin 81 (1963) $711-713$.

Radbruch, R.: Rechtsphilosophie, 3. Aufl. Leipzig 1932/4,-8. Aufl. Stuttgart 1950-1973: zitiert nach Kaufmann. A.. Seite 128 u. 154 .

Ritter, H.: Die Rolle der Anthropologie im NS-Staat. In: J. Peiffer: Menschenverachtung und Opportunismus. Attempto Verlag. Tübingen 1992.

Rothmaler, C.: Zwangssterilisation nach dem „Gesetz zur Verhütung erbkranken Nachwuchses". Deutsches Ärzteblatt 86 (1989) B-123-126.

Schüller, B.: Die Begründung sittlicher Urteile. Typen ethischer Argumentation in der Moraltheologie. Patmos Verlag, Düsseldorf (1980).

Schmuhl, H.-W: Rassenhygiene, Nationalsozialismus, Euthanasie: von der Verhütung zur Vernichtung .,lebensunwerten Lebens", 1890-1945. Vandenhoeck u. Ruprecht, Göttingen (1987).

Stauber, M., G. Kindermann: Ober inhumane Praktiken der Frauenheilkunde im Nationalsozialismus und ihre Opfer, Geburtsh. und Frauenheilk. 54 (1994) $479-488$.

Tauber, A. I.: Science, technology, and the art of medicine. N. Engl. J. Med. 329 (1993) 1747-1748.

VanValen, L. M.: A cost of progress. Nature 339 (1989) 248.

Wuttke, W.: Ideologien der NS Medizin. In Peiffer, J.: Menschenverachtung und Opportunismus. Attempto Verlag, Tübingen (1992). 


\section{Anhang I}

(E. Kuss, „offener Brief" vom 23.12.1991)

Liebe Kolleginnen und Kollegen, nachdem ich in der Klinikbesprechung am 21. Dezember den Vortrag - Weihnachten 1916 - Weihnachten 1991,75 Jahre .Maistraße“ - diskutiert hatte, wurde ich als .. Ewiggestriger" eingeordnet. Damit Sie nachweislich erkennen können, was als .Ewiggestrig“ bezeichnet wurde, präzisiere und fixiere ich meinen Diskussionsbeitrag wie folgt.

1. Ich distanzierte mich von der Form der öffentlichen Verurteilung des ehemaligen Direktors unserer Klinik, Professor Dr. H. Eymer. Die Verurteilung, die, zumindest nachträglich, auch vom jetzigen Direktor dieser Klinik ausdrücklich gebilligt worden ist, hatte ihren spezifischen Ausdruck darin gefunden, da $B$ im Rahmen der Adventsfeier dieser Klinik das Bild des zur Wand umgedrehten Kopfes des ehemaligen Direktors der Klinik gezeigt wurde. Den Begleittext, u.a. die Begründung dieser Position, sprach nicht irgendwer, sondern der Leitende Oberarzt dieser Klinik.

2. Ich richtete meine Aussagen gegen den Inhalt des Vortrages, soweit er die undifferenzierte Anklage und Verurteilung eines Mannes betraf, der in der Zeit des Nationalsozialismus Direktor der 1. Frauenklinik der Universität in der „Hauptstadt der Bewegung“ war und auch in der Zeit danach. Undifferenziert deswegen, weil Anklage und Verurteilung von einer unabgeschlossenen Doktorarbeit eines Laien der Medizinhistorik abgeleitet worden waren und weil die Möglichkeit der argumentativen Auseinandersetzung mit der Anklage nicht eingeräumt worden war. Auch Mitscherlich und Mielke sahen ihre historische Aufgabe nicht darin, ,,irgend jemanden in den Augen seiner Mitmenschen anklägerisch zu belasten,..." und ..Wir haben an allen Stellen auch die Argumente, welche die Angeklagten zu ihrer Verteidigung vorbrachten, mit aufgenommen" (in: Medizin ohne Menschlichkeit, 1960). Eine Darstellung und Analyse der Geschichte auch unserer Klinik nach Prinzipien der Historik habe ich ausdrücklich begrüßt.

3 . Ich wandte mich gegen die Selbstgefälligkeit in der heutzutage billig erhältlichen Verurteilung der in der Vergangenheit „nach ihren Normen und nicht nach unserer Weisheit" Handelnden (Nipperdey. Th.: Nachdenken über die deutsche Geschichte. 1986). Im vorliegenden Fall war es die kraft Amt maßgebliche Beteiligung Eymers an der zeitgemäßen, nicht zwingend NS-abhängigen. Sterilisation „Erbkranker“. Diese erfolgte auf der Grundlage des weitgehend gebilligten .,Gesetzes zur Verhütung erbkranken Nachwuchses" nach Beschluß von Erbgesundheitsgerichten (..Ein ähnlicher Gesetzentwurf war bereits in der Weimarer Republik von Experten vorbereitet worden.": Müller-Hill, B.: Tödliche Wissenschaft, 1984): ("... the sterilization law of 1933 which led to the sterilization of some 350000 to 400000 persons, was at first welcomed internationally ...": Nature $334,573,1988)$; ("... the sad saga of the involuntary sterilization, between 1907 and the early 1960s, of about 60000 " "mental defectives". a broad term that includes retarded persons, criminals. prostitutes, rapists, epileptics, inebriates, and the insane. The aim of this surgical campaign was to purify the population of the United States and to improve its ge- netic pool." New Engl. J. Med. 325,1386, 1991); (s. a. Rothmaler, C.. Deutsches Ärzteblatt 86, B-123, 1989).

Eymer handelte somit legal und nach ethischen Werten, die von der Gesellschaft seiner Zeit weitgehend akzeptiert waren, allerdings nach wissenschaftlichen Erkenntnissen, die sich nach heutigem Stand der Humangenetik als defizitär erwiesen haben.

Zur Werteordnung: Im Adventsvortrag des Leitenden Oberarztes wurde antithetisch zu Eymers-Haltung die Haltung der Vinzentinerinnen gerühmt. Aber, diese Anti-Eugenik-Haltung der katholischen Kirche war von der gleichen Werteordnung abgeleitet wie es ihre Haltung zu Sexualität, Verhütung, Abtreibung ist. Dieser integrale Teil dieser Werteordnung jedoch wird in den von uns zu verantwortenden Klinikbesprechungen nicht gerühmt. Was aber ist unsere Werteordnung? Wie stringent ist sie?

Zur wissenschaftlichen defizitären Grundlage der Eugenik: Die Eugenik der zwanziger und dreißiger Jahre war ähnlich schwach fundiert wie es heute noch z. B. Psychotherapie und Psychosomatik sind. Auch die radikale Operation und die aggressive Chemotherapie der Krebspatienten und vieles andere von dem, was Ärzte heute und hier mehr oder weniger unreflektiert ordinieren, ist nicht über jeden Zweifel erhaben. Zu lernen ist also von damals für heute die Notwendigkeit wissenschaftlich-skeptischer Haltung auch und besonders zu dem, was in der Medizin gerade Mode ist. Die Tatsache aber, da $B$ wir zur fachgerechten Diskussion von Problemen dieser Art, zur Diskussion wissenschaftlicher Arbeiten der Klinik und der Grundlagen unseres Faches und ihrer metawissenschaftlichen Implikationen, kaum eine Stunde im Jahr ansetzen, sollte uns veranlassen, eine selbstgefällige Verurteilung unserer Vorgänger und Beurteilung vermeintlich eigener Leistungen $\mathrm{zu}$ überdenken. Gegen Defizite in Forschung und Lehre und für manche anderen Probleme ärztlicher Zusammenarbeit ..Weihnachten 1991“ „hätten das moralische Engagement und die praktische Solidarität ihren Platz, die im Hinblick auf die historische Erscheinung des Nationalsozialismus in ihrem rationalen Kern längst zu Selbstverständlichkeiten geworden sind, so daß die Regel wissenschaftlicher Darstellung und Forschung heute wieder uneingeschränkt in Kraft treten sollten." (Nolte, E., MittHV, 1989).

Meine Ausführungen waren und sind u. a. Ausdruck einer Geschichtsauffassung, wie sie Historiker von Ranke bis Nipperdey und Nolte nachweislich vertreten, und die, wie aufgezeigt, auch Mitscherlich und Miel$k e$ respektierten. Diese Geschichtsauffassung wird aber in der Tat auch als ewig gestrig abgetan, und zwar von vermeintlich progressiven, simplifizierenden. doktrinären und somit wissenschaftsfeindlichen Ideologien wie Nationalsozialismus, Marxismus und anderen Strömungen, die Historie zur jeweiligen Agitation und Selbstdarstellung instrumentalisieren.

lch wünsche Ihnen eine akademische Atmosphäre, die Bedingung der Möglichkeit für Forschung. Lehre und ärztliche Tätigkeit in Skepsis. Selbstdisziplin und Freiheit, die zu schaffen und zu gewähren Weihnach- 
ten 1991 zu den Rechten und Pflichten der Universität und somit zu den Aufgaben ihrer Professoren und Doktoren gehört.

Ihr (gez, Erich Kuß)

\section{Anhang 2}

(E. Kuss, Deutsches Ärzteblatt 91, B-538. 1994)

\section{Leserbrief zu}

\section{Gynäkologie und Nationalsozialismus}

(Dr. Gerhard di Pol, Deutsches Ärzteblatt 90, B-2303, vom 26. November 1993)

Wenn es denn kein anderer sagt, sage ich es: die im oben genannten Artikel von Professor Dr. $M$. Stauber, vom Leitenden Oberarzt der I. Frauenklinik der Universität München festgestellte ..Verleugnung. Verdrängung. Unterdrückung und Verharmlosung“ des Nationalsozialismus in der I. Frauenklinik der Universität München gibt es dort nicht, gab es jedenfalls nicht, solange ich dort gearbeitet habe (1. August 1960 bis 30. September 1992) und sofern ich den Partialaspekt ..Unterdrückung des Nationalsozialismus" als Ausdruck des Öbereifers außer acht lasse. Als gelernter Wissenschaftler kann ich nicht umhin, selbst diese Behauptung, diese Zurückweisung einer absurden Unterstellung, zu belegen: J. Zander, 1970-1987 Direktor der inkriminierten Klinik, schrieb zum einschlägigen Thema u. a. .,Milestones in Gynecology and Obstetrics - on the Occasion of the Centenary of the German Society of Gynecology and Obstetrics" (1. Special Lecture, XI World Congress. International Federation of Gynecology and Obstetrics, Berlin, 16. September 1985; Deutsche Übersetzung in: Zur Geschichte der Gynäkologie und Geburtshilfe, L. Beck (Hrsg.), Springer-Verlag, Heidelberg usw. 1986) und „Ärzte im Zwielicht. Medizin zwischen totalitärer Ideologie und Individualethik" (in: Der 2. Weltkrieg und die Gesellschaft in Deutschland 50 Jahre danach, Venanz Schubert (Hrsg.). Eos Verlag Erzabtei St. Otilien 1992) und diskutierte damit zusammenhängende Fragen nachweislich mit zahlreichen Kollegen und Interessenten innerhalb und außerhalb der Klinik. Dem zusätzlichen Vorwurf Staubers, dem Vorwurf der „Unzureichenden Aufarbeitung" in dieser Klinik kann ich nicht widersprechen. Nur: kein Mensch, auch Stauber nicht. wird allgemeinverbindlich feststellen können, was denn .Zureichende Aufarbeitung“ ist. Möglicherweise kann aber allgemeinverbindlich festgestellt werden, was ..Zureichende Aufarbeitung" nicht ist.

.Zureichende Aufarbeitung " ist es sicher nicht, wenn, wie im oben genannten Artikel, der Berichterstatter die gegenwärtigen Realitäten und die historischen Gegebenheiten der I. Frauenklinik der Universität München nicht so sehen und beschreiben kann oder will. wie sie tatsächlich sind, waren und wurden. .Zureichende Aufarbeitung" ist es sicher nicht. wenn, wie im oben genannten Artikel, der Berichterstatter ein Lippenbekenntnis zur ..Verurteilungsabstinenz" ablegt, um sich dann zu Lasten lebender oder toter Kollegen zu profilieren. Möglicherweise verstellt ihm Voreingenommenheit den Blick auf gegenwärtige und vergangene Ereignisse und verhindert differenzierte Wahrnehmung dessen, was ist und was war. Wer zahlreiche gegenteilige Aussagen bewußt vernachlässigt und in Kenntnis der AuschwitzVerbrechen einem toten Kollegen lapidar unterstellt, daB er ,.völlig die Rassenideologie der Nationalsozialisten“ vertrat, verläßt, von anderem abgesehen, die Grundlagen empirisch-wissenschaftlicher Historiographie. Wer nebulös aber vermutlich vorwurfsvoll die .Übernahme bestimmter Wertvorstellungen" beklagt ohne darzulegen, welche Wertvorstellungen ihn und seine Vorstellung von Psychosomatik leiten und vor zukünftiger ..Verurteilungsabstinenz" schützen, mag die Wertvorstellungen empirischer Wissenschaft gering schätzen. Die an Historie und damit an unseren geistigen Grundlagen und Entwicklungen oder an "Aufarbeitung" interessierten Leser des Deutschen Ärzteblattes wurden früher schon besser informiert, z. B. mit den einschlägigen Artikeln ..Medizin im Nationalsozialismus" von $G$. Baader , Rassenhygiene und Eugenik" (Deutsches Ärzteblatt 85, B1357-1360 [1988]), .Zwangssterilisation nach dem .,Gesetz zur Verhütung erbkranken Nachwuchses “" von $C$. Rothmaler (Deutsches Ärtzeblatt 86, B 123-B 126 [1989]) oder auch mit der einschlägigen Passage im Leserbrief .Angelastete Schuld“ von G. Huwer (Deutsches Ärzteblatt 84, B-1452-1454 [1987]).

Prof. Dr. Dr. E. Kuss

Ortolfstraße 15

81247 München 